\title{
Exploring a Quantum Hebbian Model of Feature Map Formation
}

\author{
Priti Gupta and C.M. Markan \\ Department of Physics and Computer Science, Dayalbagh Educational Institute, \\ Agra, India \\ \{gupta.priti.84,cm.markan\}@gmail.com
}

\begin{abstract}
The brain self-organizes into feature maps or neural assemblies on receiving inputs. Similar self-organization is possible in artificial systems only if the principles that the brain employs are exploited. Existing models explaining feature map formation cover only some aspects of local feature map formation. It is unlikely that the brain employs different mechanisms to form local and global feature maps and hence there is a need to explore a single mechanism that could account for neural interactions at all levels. If we take the brain to coexist as a quantum and a classical system, certain insights can be obtained about neural development. In this paper we explore a quantum hebbian model of interaction between the quantum and classical processes in the brain, which in synergy with mental force of directed attention, seems to have the potential to explain the formation of both local and global feature maps. Introducing this duality also helps us address higher level issues like mind wandering, zombie modes, volition etc.
\end{abstract}

\section{Introduction}

The brain's cortical structure adapts to environmental inputs and hardwires itself during a critical learning period [15]. If we can have machines that adapt to the environment in a similar way, we move a step closer to having truly intelligent machines. The brain organizes itself into feature maps in response to the inputs it receives. If we can replicate such feature maps in hardware, it could have wide applications in robotics, artificial sensory systems and neural prosthetics. Recently, it has been shown that analogue hardware can be fine-tuned to work in the environment for which it is designed by introducing field programmability 7. While technological advances have paved the way for us to build neuromorphic hardware that can emulate feature map formation [3], what is important now is to understand in depth how global feature maps are formed in the brain. For simplification feature maps can be broadly classified into two types: local and global feature maps. While local feature maps represent the connectivity patterns within a localized cortical area e.g. visual, auditory etc., global feature maps represent the connectivity patterns across spatially separated cortical areas. Existing models 2] assume prior interconnectivity in the form of Mexican Hat connections. This kind of precise connectivity is not found in a developing 
brain. Other models, based on non-axonal means of communication e.g. Diffusive Hebbian 9] etc, overcome this limitation, but they are confined to intra-modality or local interaction. It is unlikely that the brain employs different mechanisms for forming local and global connections and therefore this problem needs reexamination. Neural connections are formed by the famous Hebb's Rule 'Cells that fire together wire together' and Stent's modification 'Cells that do not fire together compete for elimination' i.e. if the activity of two neurons is synchronized, synaptic connection joining them strengthens whereas if the activity is not synchronized this strength decreases and finally the connection is eliminated. To understand how inter-cortical or global neural assemblies are formed, we need to decipher how in the absence of any direct physical connections, spatially separated neurons synchronize (with zero-lag)? This is a paradoxical situation that we term as the Neural Development Paradox (NDP). In order to understand how global feature maps are formed we need to break the NDP.

\section{Need for a Quantum Mechanical Approach}

The motivation to use quantum mechanics to unravel some of the mysteries of the brain stems from many reasons. Biological systems are made up of molecules and atoms and the most advanced theory that explains the interaction between them is quantum theory. Recently, quantum effects have been shown to operate at high temperatures in biological systems e.g. in birds vision, photosynthesis, olfaction [8] and many experiments are being performed to find out if they exist in the brain [5]. The brain processes various sensory inputs e.g. visual, auditory etc. at different times and locations, even then we perceive them as simultaneous events. Since synaptic transmission and axonal transfer of nerve impulses are too slow to organize coordinated activity in large areas of the brain, other nonaxonal means need to be explored. In a recent theory, Hameroff et.al. [4, 5] suggest that Microtubules inside cells support long-range quantum coherence, enabling quantum information processing to take place at the sub-cellular level. Cells interconnected by gap junctions form networks which fire synchronously, behaving like one giant neuron [6], and possibly accounting for synchronized neural activity such as coherent $40 \mathrm{~Hz}$ waves, and could be a possible solution to the NDP.

\section{Formation of Neural Assemblies and Quantum Zeno Effect}

The formation of neural assemblies is based on three events. First, for a particular stimulus, a single neural assembly is activated from all possible combinations of neural assemblies in the brain. Second, there is sustained synchronous activity of the neurons in that assembly, and third, there is change in the synaptic weights connecting its neurons, so that they become hardwired. All these events can be explained in quantum mechanical parlance by considering Quantum Entanglement, Quantum Zeno Effect (QZE) [1] and Quantum Decoherence. QZE 
states that we can maintain the state of a system as long as we perform measurement on it. According to Penrose-Hameroffs quantum approach, the subneuronal microtubules form a quantum computer whose collapsed states are conscious experiences manifesting in the form of an activated neural assembly. Quantum Entanglement among the sub neuronal microtubules of neurons [4] are responsible for the selection of a group of neurons forming a neural assembly. The neurons whose microtubules are entangled get connected by gap junctions to form one large hyper-neuron. When the quantum wave function collapses or de-coherence occurs, after a critical threshold has been reached [1], the neurons in the neural assembly fire in synchrony. Quantum Zeno Effect sustains this synchronous activity by repeated collapses to the same state of the system so that hebbian learning can strengthen the synaptic connections. The question is what causes the QZE? The role of attention in the learning process has always been acknowledged. The relation between attention and QZE can be seen in [13, 14] and it seems reasonable to establish that attention causes the Quantum Zeno effect to maintain the brain in a particular state and facilitates neuro-plastic changes.

\section{Quantum Neural Computation}

As a preliminary effort towards understanding how quantum computation in the brain works, we build a basic unit called a Quantum Neural Computer (QNC). Previous efforts to combine the fields of Quantum Computing and Artificial Neural Networks [12] have been made to enhance the computational power of ANNs, but none has been used with a view to understand brain function. In our model, the function of the QNC is analogous to the microtubule cytoskeleton quantum computer. The QNC takes $\mathrm{N}$ inputs that are either $\mid 0>$ or $\mid 1>$ and transforms them to an output state $u=\alpha|0>+\beta| 1>$ where $\alpha$ and $\beta$ are derived from the weights and $\alpha^{2}$ and $\beta^{2}$ are the probabilities that the output $u$ will be a $\mid 0>$ or $a \mid 1>$. The unitary transformation is implemented by a weight matrix described in [10] given as:

$$
W_{i}=\sum_{x=0}^{2^{N}-1} c_{x}^{i}(x) \quad \text { where } c_{x}^{i} \epsilon\{0,1\} \text { and } x \in I
$$

For the sake of normalization, only one $c_{x}^{i}$ is 1 for a particular input, the rest are 0 . The $\alpha$ and $\beta$ are computed as below.

$$
\alpha=\sum_{i} \delta_{a_{i, 0}} W_{i}, \quad \beta=\sum_{i} \delta_{a_{i, 1}} W_{i} \quad \text { and } \quad \alpha^{2}+\beta^{2}=1
$$

There are $2^{3} \times 2^{3} \times 2^{3}$ possible weight matrices. The strength of quantum computation lies in these many possibilities of transformations and this is what we harness in our model. The QNC computes all possible output states, transforming the inputs over all possible weight matrices simultaneously, by means of superposition. On collapse, the system reduces to a single output state that is chosen randomly from the set of all possibilities. 


\section{Quantum Hebbian Learning}

Every Quantum collapse leads to increment in knowledge 14. In the human brain, the modification in synaptic weights accounts for accumulation of knowledge. In order to understand how classical synaptic weights can be affected by quantum collapses we need to model their interaction. Let $\mathrm{C}$ be a certain classical weight, $\delta$ be the classical learning rate (representing the classical accumulation of knowledge for every quantum collapse), and Q to be the impact of the current state of QNC. $\delta$ is a very small value (say .001). By repeated collapses to the same state by means of attention and QZE, the classical learning becomes sufficiently large, causing a permanent change in the synaptic weights. The equation below describes the above interaction.

$$
\text { Next Collapsed State } \leftarrow \alpha Q+(1-|\alpha Q|)^{*} C
$$

Where, $C=C+\delta Q, \alpha$ : attention parameter, $\delta$ : classical learning rate, $Q$ : current state of the $\mathrm{QNC}, \alpha Q$ : qualia, $C$ : classical weight. Here the first part of the equation $\alpha Q$ determines the quantum influence and the term $(1-|\alpha Q|)^{*} C$ determines the classical influence on the next collapsed state. Depending on the values of the various parameters that are involved in this equation, many interesting situations arise(see table 1).

Mind Wandering: The absence of attention $(\alpha=0)$ and when the brain is not developed (i.e. $\mathrm{C}$ is very small), leads to a state where all collapses of the QNC are random (determined by the quantum influence). This is analogous to the state of mind wandering.

Table 1. (Here, $\beta=(1-(|\alpha Q|))$

\begin{tabular}{ccc}
\hline & $\begin{array}{c}\text { Non Developed Brain } \\
(C \text { is low })\end{array}$ & $\begin{array}{c}\text { Developed Brain } \\
(C \text { is high })\end{array}$ \\
\hline $\begin{array}{c}\text { No Attention } \\
(\alpha=0)\end{array}$ & Mind Wandering & Zombie \\
\hline $\begin{array}{c}\text { Full Attention } \\
(\alpha=1)\end{array}$ & $\begin{array}{c}\text { Quantum Zeno } \\
(\text { when } \alpha \mathrm{Q}>\beta \mathrm{C})\end{array}$ & $\begin{array}{c}\text { Volitional Choice } \\
(\text { when } \alpha \mathrm{Q}>\beta \mathrm{C})\end{array}$ \\
\hline & $\begin{array}{c}\text { Mind Wandering } \\
(\text { when } \alpha \mathrm{Q}<\beta \mathrm{C})\end{array}$ & $\begin{array}{c}\text { Zombie } \\
(\text { when } \alpha \mathrm{Q}<\beta \mathrm{C})\end{array}$ \\
\hline
\end{tabular}

Zombie: In absence of attention $(\alpha=0)$, when the brain is developed $(\mathrm{C}$ is large), the classical accumulated knowledge determines the state to which the subsequent collapse happens e.g. when youre driving a car in an auto-pilot mode without paying attention to details. 
Quantum Zeno: In the presence of attention $(\alpha=1)$ and when the classical weights have not developed i.e. $\alpha \mathrm{Q}>\beta \mathrm{C}$, by focused attention the subsequent collapses can be made to happen to the same state. The quantum part plays the main role. In this state accumulation of knowledge takes place, $\mathrm{C}=\mathrm{C}+\delta \mathrm{Q}$. When $\mathrm{C}$ become sufficiently large, we say hebbian learning has strengthened the synaptic connections of the assembly.

Volitional Choice (Free Will): This is a situation that arises when even though the classical weights are well developed, by keeping attention high, the collapse of the QNC can be made to be determined by the quantum part rather than the classical part. This bears analogy with the problem of volitional choice which has not been explained neurobiologically. It seems that by having a dual system (quantum + classical) in which one system has a supervisory role, some insight into how free will could be working can be obtained. A MATLAB simulation model of the Quantum Hebbian paradigm was developed for a 3 input QNC. Figure 1 shows the simulation results.
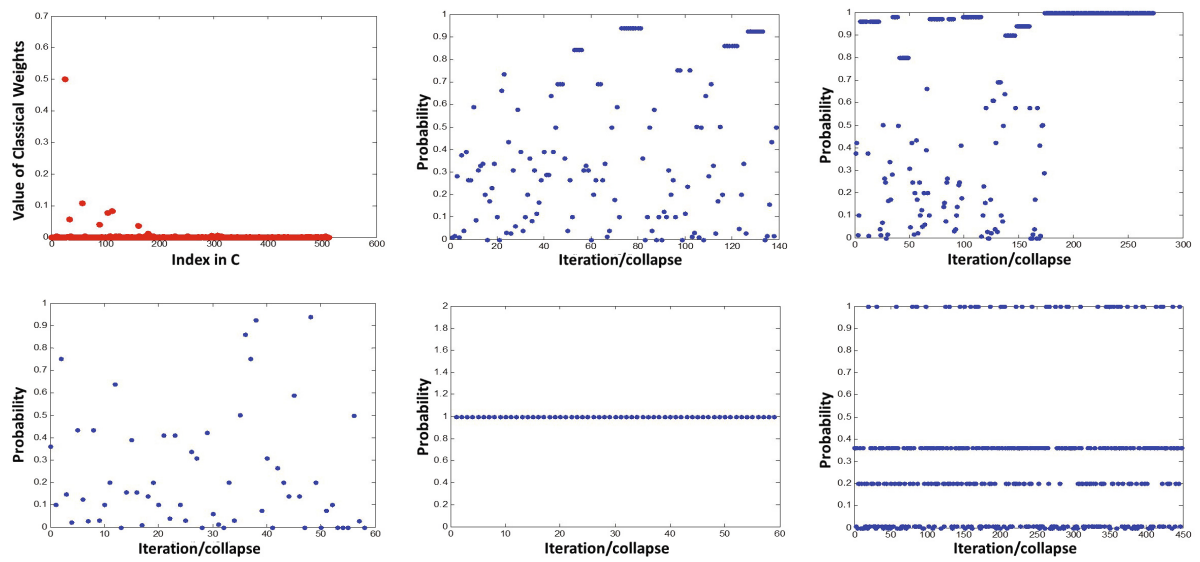

Fig. 1. [Row 1, a-c, row 2, d-f] a). Accumulation of classical weights. Out of many possibilities of neural assemblies, weights of only one neural assembly have been strengthened (the $\mathrm{x}$-axis shows the index of the classical weights and y-axis shows the value of classical weight). b). QZE, repeated collapses to the same state can be observed from the continuous dots. c). Mind wandering state $(\alpha=1)$. Collapses are mostly random, QZE too small to change classical weights permanently. d). Mind wandering state $(\alpha=0)$, the collapses are completely random. e). Shows the zombie effect, $(\alpha=0)$, the collapses are determined by the classical weights that have developed the most. f). Free will state. Here despite of the presence of a developed classical weight (dots corresponding to probability $=1$ ), majority of the collapses are happening to a state determined by the quantum part (dots corresponding to probability .35) (for figures b-f, x-axis represents the number of iteration and y-axis shows the probability) 


\section{Discussion}

A framework for quantum and classical interaction in the brain has been proposed. The Quantum Hebbian Equation not only indicates how quantum collapses could lead to classical learning but also determines to which state subsequent collapses will happen. With the attention parameter regulating the QNC, insight into the behaviour of the brain in the presence and absence of attention can be obtained. The different states of the brain like learning in presence of attention, zombie and mind wandering and volitional choice have been explained on the basis of this quantum and classical interaction. While this work is mostly exploratory in the present state, with research in quantum computing and VLSI design on the fore, it is just a matter of time that we would be able to create machines based on this kind of dual interaction of quantum and classical information.

\section{References}

1. Diosi, L.A.J.O.S.: Models for universal reduction of macroscopic quantum fluctuations. Physical Review A 40(3), 1165 (1989)

2. Erwin, E., Obermayer, K., Schulten, K.: Models of orientation and ocular dominance columns in the visual cortex: A critical comparison. Neural Computation 7(3), 425-468 (1995)

3. Gupta, P., Bansal, M., Markan, C.M.: Neuromorphic Adaptable Ocular dominance maps. In: Ghosh, A., De, R.K., Pal, S.K. (eds.) PReMI 2007. LNCS, vol. 4815, pp. 49-56. Springer, Heidelberg (2007)

4. Hameroff, S.R.: Quantum coherence in MTs: a neural basis for emergent consciousness? Journal of Consciousness Studies 1, 98-118 (1994)

5. Hameroff, S., Tuszynski, J.: Quantum states in proteins and protein assemblies: The essence of life? In: Proceedings of SPIE Conference on Fluctuationa and Noise, Canary Islands (2004)

6. Kandel, E.R., Siegelbaum, S.A.K., Schwartz, J.H.: Synaptic transmission. In: Kandel, E.R., Schwartz, J.H. (eds.) Principles of Neural Science, 3rd edn., pp. 121-134. Elsevier, New York (1991)

7. Kapur, G., Mittal, S., Markan, C.M., Pyara, V.P.: A Unique Design methodology to generate reconfigurable Analog ICs with simplified Design Cycle. In: Workshop on Unique Chips and Systems UCAS-7, p. 28 (2012)

8. Lambert, N., Chen, Y.N., Cheng, Y.C., Li, C.M., Chen, Y.G., Nori, F.: Quantum Biology. Nature Physics 9, 10-18 (2012)

9. Markan, C.M., Bhaumik, B.: A Diffusive-Hebbian model for cortical orientation maps formation. In: Proc. of IJCNN 1999, Washington DC (1999)

10. Miranker, W.L.: Quantum Neurons. Tech report, Univ. of Yale, Yale/DCS/tr1234 (2002)

11. Misra, B., Sudarshan, E.C.G.: The Zeno's paradox in quantum theory. Journal of Mathematical Physics 18, 756 (1977)

12. Rudolph, T.G., Jensen, H.J.: A Heuristic Review of Quantum Neural Networks (2011)

13. Schwartz, J.M., Begley, S.: The mind and the brain. HarperCollins e-books (2009)

14. Stapp, H.P.: Mind, matter and quantum mechanics. Springer, Berlin (1993)

15. Sur, M., Leamy, C.A.: Development and Plasticity of Cortical Areas and Networks. Nature 2, 251-262 (2001) 